\title{
Editorial for the MEDIA Special Issue on MICCAI 2012
}

This issue of Medical Image Analysis presents a selection of the best work presented at MICCAI 2012 in Nice. We are extremely grateful for the interest that MedIA has shown in supporting MICCAI over the years, and in the way this journal has complemented the Society in promoting the excellence of the Medical Image Computing and Computer Assisted Intervention arms of the MICCAI society.

For this issue, full-length papers were solicited from the authors of the award winning and top scoring submissions at the conference. These papers went through the standard MedIA review process of obtaining two or three peer reviews. From the 16 papers originally invited, the 11 papers appearing here made it successfully through the review process. A 12th paper entitled "Reliable estimation of incoherent motion parametric maps from diffusion-weighted MRI using fusion bootstrap moves" by Freiman et al. appeared in the issue of April 2013 (Volume 17, Issue 3) of the Medical Image Analysis journal.

We have grouped the selected papers into the three themes of Image-Guided Intervention, Image Analysis, and Diffusion Imaging.

The Image-Guided Intervention section contains three papers. Kwitt et al. tackle the problem of detecting objects of interest in ultrasound video sequences acquired with low-cost ultrasound devices. Lasser et al. proposes an optimization of the position of mobile freehand nuclear imaging detectors in an intra-operative setting to decrease acquisition time. Zapella et al. attempt to automatically classify surgical gestures from video and kinematic data in order to establish objective criteria for surgical skills assessment.

The Image Analysis section includes six papers. Su et al. present a semi supervised clustering approach based on phase retardation features for segmentation of phase contrast microscopic images of cells. Iglesias et al. explore the integration of uncertainty over free parameters in a segmentation algorithm in order to estimate confidence intervals for hippocampal subfields volume estimates extracted from brain MRI scans. Shi et al. introduce a sparsity constraint in the parameter space to improve robustness and accuracy when temporally registering image sequences with a free-form deformation method. Konukoglu et al. aim to compute nearest neighbors between annotated medical image databases. They introduce a supervised learning algorithm that operates on user-defined distances that may include semantic information. Gibson et al. examine the issue of finding the maximum registration error or the number of subjects required to validate a novel imaging modality when comparing it to an accepted reference standard modality. Marchesseau et al. study the estimated cardiac biophysical parameters of healthy and pathological cases based on a personalized electromechanical model of the heart in cine MR images. 
The Diffusion Imaging section contains two papers. Merlet et al. introduce a parametric dictionary learning approach to recover the diffusion signal and its main features with a reduced number of measurements. Côté et al. propose an online validation system for tractography processing pipelines and draw interesting conclusions about the impact of different diffusion acquisition settings, local estimation and tracking methods.

We trust that this special issue will provide a comprehensive snapshot of the best works at MICCAI 2012 and help attract high quality submissions to the MICCAI conference series.

Guest Editors

Hervé Delingette

Polina Golland

Kensaku Mori 